\title{
IDENTITY OF CONIOPTERYX MADAGASCARIENSIS MEINANDER, 1974 (NEUROPTERA: CONIOPTERYGIDAE), WITH DESCRIPTION OF THREE NEW SPECIES
}

\author{
GYÖRGY SZIRÁKI \\ Department of Zoology, Hungarian Natural History Museum \\ H-1088 Budapest, Baross utca 13, Hungary; E-mail: sziraki@zoo.zoo.nhmus.hu
}

\begin{abstract}
Investigation of a large coniopterygid material from Madagascar showed that Coniopteryx (Coniopteryx) madagascariensis Meinander, 1974 is not conspecific with the forms that were described and illustrated later as variations of this insect. This statement was supported also by the examination of the holotype of C. madagascariensis. Coniopteryx (Coniopteryx) angusticauda sp. n., Coniopteryx (C.) evellana sp. n. and Coniopteryx (C.) malgasensis sp. n. are described as new species. These are referring the three different forms, which were erroneously handled as C. madagascariensis. All the three new species belong to C. lobifrons species group, which hithero was known only from the Oriental Region.
\end{abstract}

Key words: Coniopterygidae, Madagascar, identity of species, new species, Oriental Region.

\section{INTRODUCTION}

The well illustrated original description of Coniopteryx madagascariensis - the first Coniopteryx species from Madagascar - was published about forty years ago (Meinander 1974). Later on an additional description was given, with figures of three forms of male genitalia as variations of $C$. madagascariensis (Meinander 1998). Differences between the three forms, as well as dissimilarity between these and the genitalia figured in the original description were surprisingly large - much larger than in other known cases in the family Coniopterygidae. Moreover, setose outgrowths were on the pedicel and first flagellar segment of the newly examined specimens, and there was a flattened projection between their antennae, which features were not mentioned in the original description of $C$. madagascariensis. In connection of this significant discrepancy it was stated that "presence of a digitate apophysis on the vertex between the antennae of the male... is a character that was overlooked when I described this species" (Meinander 1998). Recently I have received a very large coniopterygid material from Dr. Norman Penny, California Academy of Sciences (CAS). This material contained specimens of all the three forms, which was regarded by MeINANDER (1998) as C. madagascariensis, as well as a specimen agreeing with the original description of this species. Besides, I have the possibility to examine the holotype of $C$. madagascariensis. (The females of the investigated Madagascan material were identifiable only at genus level. These are not handled in present paper.) 


\section{MATERIAL AND METHODS}

Apart from the holotype of the Coniopteryx madagascariensis Meinander, 1974, which was borrowed from the Muséum National d'Histoire Naturelle (Paris), the examined material was collected in different parts of Madagascar in the framework of the Madagascar Project of CAS. Vast majority of the coniopterygid specimens was captured by Malaise traps. The names of collectors are given in the following abbreviations: $\mathrm{ES}=$ Evert I. Schlinger, F = Brian Fischer, G = Charles E. Griswold, HH = Rasolondalao Harin'Hala, I = Michael Irwin, JR = Jean S. Randrianarisoa, JS = Jere Schweikert, K = David H. Kavanaugh, $\mathrm{MR}=$ Marie J. Raherilalao, $\mathrm{P}=$ Norman D. Penny, $\mathrm{U}=$ Darell Ubick. Holotypes and most of the paratypes of the new species are deposited in collection of California Academy of Sciences (CAS). Paratype of $C$. angusticauda sp. n. and a few paratypes of $C$. evellana sp. $n$. and C. malgasensis sp. n. are housed in the Hungarian Natural History Museum (HNHM).

\section{TAXONOMY}

\section{Coniopteryx (Coniopteryx) madagascariensis Meinander, 1974}

(Fig. 1)

Examined material-Holotype: male, „,Madagascar-Centre”, Plateau Soaindrana, 2060 m. a.s.l., 14-17. I. 1958, leg.: B. R. Stuckenberg, deposited in Museum National d'Histoire Naturelle, Paris; other material: 1 male: Madagascar, Fianarantsoa Province, Ranomafana National Park, radio tower, $21^{\circ} 15^{\prime} 30^{\prime \prime}$ S, $47^{\circ} 24^{\prime} 26^{\prime \prime}$ E, $1130 \mathrm{~m}$ a.s.l., mixed tropical forest, 15-21. XII. 2001, Malaise trap, leg.: HH.

The holotype specimen is identical in all details (including the absence of a projection between the antennae) with the text and figures of the original description of the species. The specimen collected in 2001 in Ranomafana National Park agrees well with the holotype, with only an indistinct difference: in this case the endings of processus terminalis slightly tapering caudally (Fig. 1).

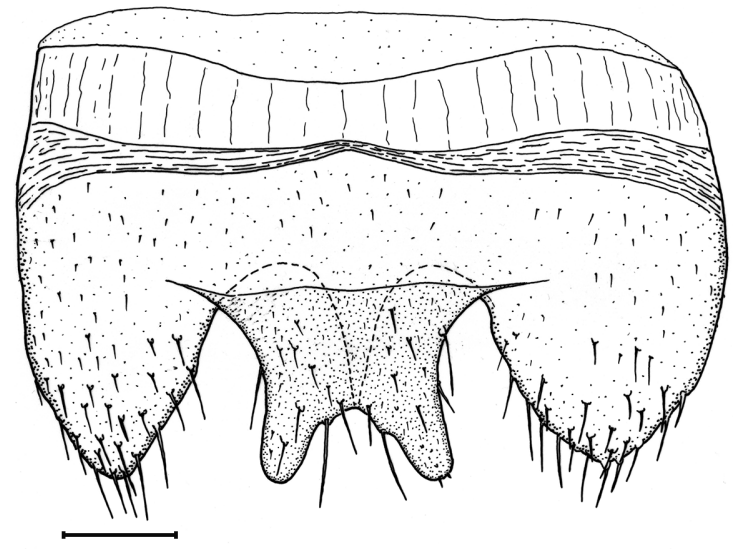

Fig. 1. Coniopteryx (C.) madagascariensis Meinander: hypandrium, ventral view. Scale: $0.04 \mathrm{~mm}$ 


\section{Coniopteryx (Coniopteryx) angusticauda sp. $\mathrm{n}$.}

(Figs 2-7)

Coniopteryx (C.) madagascariensis sensu Meinander 1998, Figs 16A-B, 17C.

Examined material - Holotype: male, Madagascar, Fianarantsoa Province, Ranomafa-

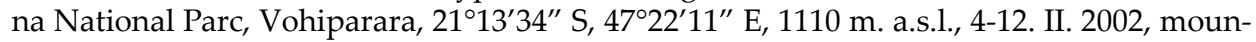
tain rainforest, Malaise trap, leg.: $\mathrm{HH}$; deposited in the collection of CAS. Paratype: male, Madagascar, Fianarantsoa Province, Ranomafana National Park, Belle Vue at Talatakely, $21^{\circ} 15^{\prime} 59^{\prime \prime}$ S, $47^{\circ} 25^{\prime} 13^{\prime \prime}$ E, 1020 m. a.s.l., 21-28. I. 2002, secondary tropical forest, Malaise trap, leg.: $\mathrm{HH}$; housed in the Hungarian Natural History Museum, Budapest.

Diagnosis: Small bodied coniopterygid, with flattened projection between the antennae. First flagellar segment with a prominent setose outgrowth. Processus terminalis narrow even at its base and strongly tapering caudally, with a small median incision.

Description: Length of the body 1.4-1.5 mm. Head capsule and palpi light brown. The anterior edge of vertex has a prominent, laterally flattened projection (as in MeINANDER 1998, Fig. 16A). Eyes moderately large, black. Antennae 0.9-1.1 mm, 28 segmented, dark brown. Scape as long as wide, pedicel slightly longer than wide, basal flagellar segments two times, median ones 1.5 times as wide as long. Pedicel has a small, first flagellar segment a prominent outgrowth apically, with rather short setae (Fig. 2). The relatively long ordinary hairs are arranged in a rather irregular ring on the basal flagellar segments and entirely irregularly on the apical ones. Scale-like hairs situated on large part of the surface of pedicel and in a rather thin apical whorl on flagellar segments.

Legs light or medium brown, large part of thorax pale ochreous, sutures and apodemes medium brown, shoulder spots dark brown. Length of fore wing 1.7-1.8 $\mathrm{mm}$, of hind wing 1.4-1.6 mm. Wing membrane and veins light or medium brown. Pregenital part of abdomen pale ochreous.

Male terminalia (Figs 3-7) well sclerotized, dark brown. Hypandrium slightly higher than long in lateral view. Processus terminalis narrow even at its base and strongly tapering caudally, with a small median incision. A well sclerotized - in caudal view reversed bell-shaped - inner structure is connected to the dorsal surface of hypandrium. This structure is a double crest, with two laterally curved anterior horns. The dorso-caudally directed processus lateralis rather large, with long setae. Anterior apodeme of hypandrium ventrally staright and narrow, laterally wide, with a hook on its dorsal ending. Gonarcus distinctly sclerotized but its ventral apodeme rather thin. Stylus forked; its long outer branch distinctly curved forwards. Inner branch of stylus very broad, with a hole, and no ventral bridge between the two inner branches. Paramere long; its anterior part rather straight. Processus ventralis strong. Processus apicalis moderately large, with an ending curved upwards, pointed in lateral view, and it has a dorsal broadening subapically. Besides, ventral part of the caudal plate of processus apicalis continued forwards in a crest bent slightly inwards. Penis sclerite consists of two distinctly sclerotized, rather short, bent and acute rods. Dorso-caudally of penis there is a moderately chitinized, saddle-shaped additional sclerite. 

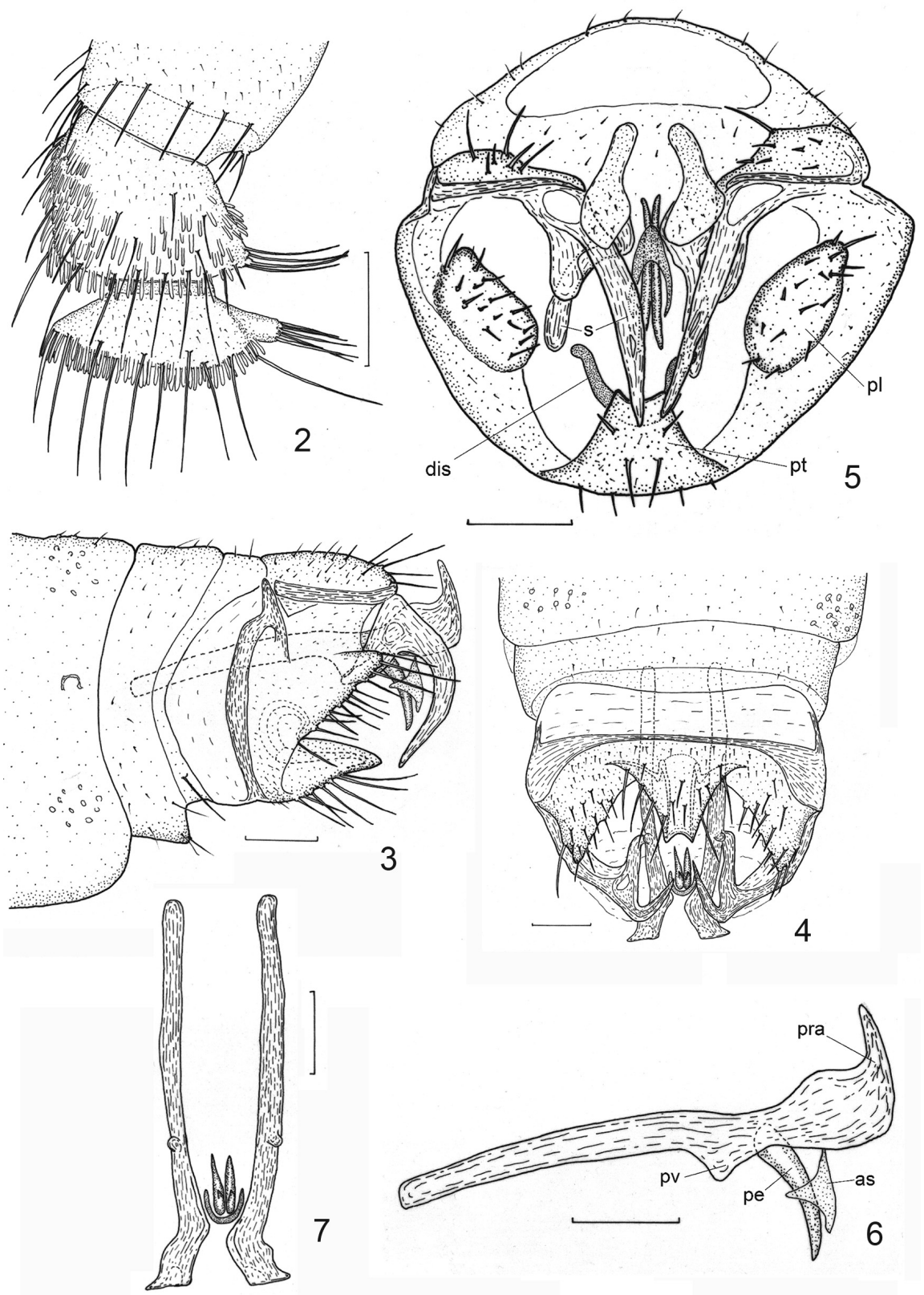

Figs 2-7. Coniopteryx (C.) angusticauda sp. n.: 2 = basal part of antenna, 3-5 = male terminalia: $3=$ lateral view, $4=$ ventral view, $5=$ caudal view, $6-7=$ paramere, penis, and additional sclerite, $6=$ lateral view, $7=$ ventral view. Abbreviations: as $=$ additional sclerite above of penis, dis $=$ dorsal inner structure of hypandrium, $\mathrm{pe}=$ penis, $\mathrm{pl}=$ processus lateralis of hypandrium, $\mathrm{pra}=$ processus apicalis of paramere, $\mathrm{pt}=$ processus terminalis of hypandrium, $\mathrm{pv}=$ processus ventralis of paramere, $\mathrm{s}=$ stylus. Scales: $0.04 \mathrm{~mm}$. 
Remarks: Coniopteryx (C.) angusticauda belongs to the Coniopteryx lobifrons group sensu SzIRÁKI (2005), and because of the narrow and caudally tapering processus terminalis, the structure of processus lateralis and the presence of an additional sclerite above the penis it resembles to Coniopteryx (C.) lobifrons Murphy et Lee, 1971.

The main distinctive features of the new species are:

- the apical whorl of the scale-like hairs on the flagellar segments of antennae is thin;

- $\quad$ outer branch of the stylus long;

- processus ventralis of paramere strong;

- $\quad$ processus apicalis of paramere with a dorsally situated subapical broadening;

- $\quad$ additional sclerite above the penis saddle-shaped.

Etymology: The new species is named after the shape of the narrow and caudally tapering hypandrium.

\section{Coniopteryx (Coniopteryx) evellana sp. $\mathrm{n}$.}

(Figs 8-13)

Coniopteryx (C.) madagascariensis sensu Meinander 1998, Figs 17B, 17E, 17G, 17I, 17K.

Examined material - Holotype: male, Madagascar, Fianarantsoa Province, Ranomafana National Park, Belle Vue at Talatakely, $21^{\circ} 15^{\prime} 59^{\prime \prime}$ S, $47^{\circ} 25^{\prime} 13^{\prime \prime}$ E, 1020 m a.s.l., secondary tropical forest, 16. X-8. XI. 2002, Malaise trap, leg.: HH; deposited in the collection of CAS. Paratypes: 3 males, Madagascar, Antanarivo Province, $3 \mathrm{~km}$ NE of Andranomay, 18 $28^{\prime} 24^{\prime \prime}$ S, 47 57' 36" E, 1300 m. a.s.l., mountain rainforest, 5-13. XII. 2000, Malaise trap, leg.: F, G et al.; 1 male, Madagascar, Antsiranana Province, Marojejy National Park, Manatenia River, $8.2 \mathrm{~km}$ NNW of Manantenia, $14^{\circ} 26^{\prime} 12^{\prime \prime}$ S, $49^{\circ} 46^{\prime} 30^{\prime \prime}$ E, $450 \mathrm{~m}$ a.s.l., rain forest, 12-25. XI. 2003, Malaise trap, leg.: F et al.; 1 male, Madagascar, Diego - Suarez Province, Montagne d'Ambre National Park, 12 ${ }^{\circ} 31^{\prime}$ S, $49^{\circ} 11^{\prime}$ E, 975 m a.s.l., 21-25. I. 2001, Malaise trap, leg.: I, ES et $\mathrm{HH} ; 1$ male, Madagascar, Fianarantsoa Province, Forêt d' Ambalagoavy nord Ikongo, Ambatombe, $21^{\circ} 49^{\prime} 39^{\prime \prime}$ S, 47 $20^{\prime} 20^{\prime \prime}$ E, 625 m. a.s.l., (day?) XI. 2000, Malaise trap, leg.: HH et I; 7 males, Madagascar, Fianarantsoa Province, Ranomafana National Parc, Belle Vue at Talatakely, $21^{\circ} 15^{\prime} 59^{\prime \prime} \mathrm{S}, 47^{\circ} 25^{\prime} 13^{\prime \prime} \mathrm{E}, 1020 \mathrm{~m}$. a.s.l., 8-15. XI. 2001, secondary tropical forest, Malaise trap, leg.: HH; 13 males, same data but 15-22. XI. 2001; 16 males, same data but 22-28. XI. 2001; 6 males, same data but 28. XI - 6. XII. 2001; 3 males, same data but 6-15. XII. 2001; 1 male, same data but 15-21. XII. 2001; 4 males, same data but 21-24. XII. 2001; 11 males, same data but 24. XII. 2001 - 2. I. 2002; 1 male, same data but 2-10. I. 2002; 2 males, same data but 10-14. I. 2002; 3 males, same data but 14-21. I. 2002; 13 males, same data but 21-28. I. 2002; 1 male, same data but 28. I. - 4. II. 2002; 2 males, same data but 12-19. II. 2002; 3 males, 19-26. III. 2002; 15 males, same data but 31. III - 7. IV. 2002; 1 male, same data but 7-14. IV. 2002; 7 males, same data but 14-23. IV. 2002; 1 male, same data but 28 . IV - 5. V. 2002; 3 males, same data but 13-23. V. 2002; 2 males, same data but 23 . V - 3. VI. 2002; 1 male, same data but 13-23. VI. 2002; 1 male, same data but 23. VI - 4. VII. 2002; 6 males, same data but 4-14. VII. 2002; 2 males, same data but 24. VII - 4. VIII. 2002; 14 males, same 
data but 16. X - 8. XI. 2002; 3 males, Madagascar, Fianarantsoa Province, Ranomafana National Parc, Jirama Water Works, $21^{\circ} 14^{\prime} 55^{\prime \prime}$ S, $47^{\circ} 27^{\prime} 08^{\prime \prime}$ E, 690 m. a.s.1., 22-28. XI. 2002, open area near a stream, Malaise trap, leg.: HH; 1 male, same data but 28. XI - 6. XII. 2001; 5 males, same data but 21-24. XII. 2001; 1 male, same data but 2-10. I. 2002; 3 males, same data but 10-14. I. 2002; 8 males, same data but 21-28. I. 2002; 1 male, same data but 16 . X-8. XI. 2002; 7 males, Madagascar, Fianarantsoa Province, Ranomafana National Park, radio tower, $21^{\circ} 15^{\prime} 30^{\prime \prime}$ S, $47^{\circ} 24^{\prime} 26^{\prime \prime}$ E, $1130 \mathrm{~m}$. a.s.l., 16. X - 8. XI. 2001, mixed tropical forest, Malaise trap, leg.: HH; 4 males, same data but 15-22. XI. 2001; 6 males, same data but 22-28. XI. 2001; 13 males, same data but 28. XI - 6. XII. 2001; 2 males, same data but 6-15. XII. 2001; 8 males, same data but 15-21. XII. 2001; 1 male, same data but 10-14. I. 2002; 15 males, same data but 14-21. I. 2002; 5 males, same data but 21-28. I. 2002; 1 male, same data but 12-19. II. 2002; 2 males, same data but 19-26. III. 2002; 2 males, same data but 26-31. III. 2002; 3 males, same data but 16-23. IV. 2002; 1 male, same data but 23-30. IV. 2002; 2 males, same data but 24 . V -4 . VI. 2002; 5 males, same data but 14-24. VI. 2002; 4 males, same data but 15-25. VII. 2002; 1 male, same data but 18-27. II. 2003; 9 males, Madagascar, Fianarantsoa

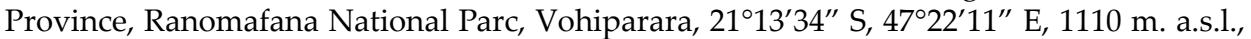
22-28. XI. 2001, mountain rainforest, Malaise trap, leg.: HH; 36 males, same data but 28. XI - 6. XII. 2001; 2 males, same data but 6-15. XII. 2001; 2 males, same data but 15-21. XII. 2001; 4 males, same data but 24. XII. 2001 - 2. I. 2002; 1 male, same data but 10-14. I. 2002; 6 males, same data but 14-21. I. 2002; 3 males, same data but 21-28. I. 2002; 33 males, same data but 28. I - 4. II. 2002; 1 male, same data but 4-12. II. 2002; 1 male, same data but 12-19. II. 2002; 1 male, same data but 21-28. II. 2002; 1 male, same data but 12-19. III. 2002; 1 male, same data but 15-22. IV. 2002; 6 males, same data but 22-29. IV. 2002; 3 males, same data but 15-25. V. 2002; 3 males, 4-14. VI. 2002; 2 males, same data but 26. VI - 5. VII. 2002; 2 males, same data but 5-15. VII. 2002; 7 males, same data but 15-25. VII. 2002; 9 males, same data but 25. VII - 3. VIII. 2002. Majority of paratypes are deposited in the collection of California Academy of Sciences, while 6 paratype specimens are housed in the Hungarian Natural History Museum, Budapest.

Diagnosis: Small bodied coniopterygid, with flattened projection between the antennae. First flagellar segment with an outstanding setose outgrowth. Processus terminalis truncated obliquely in ventral view. Median incision consists of a very shallow " $\mathrm{v}$ " shaped part and of a narrow engraving in the middle of it.

Description: Length of the body 1.3-1.8 mm. Head capsule medium brown, palpi light brown. Between the antennae the anterior edge of vertex has a prominent, laterally flattened projection. Eyes moderately large, black. Antennae dark brown, 0.9-1.2 mm, 26-29 segmented. Scape slightly shorter than wide, pedicel about as long as wide, flagellar segments 2.4-3 times as wide as long. Pedicel has a very small, while the first flagellar segment has an outstanding outgrowth apically, with rather short setae (Fig. 8). Ordinary hairs are arranged in two rather irregular rings on flagellar segments. Scale-like hairs on the whole surface of the pedicel, and in a very dense whorl on the apical half of the flagellar segments.

Legs, wings and the large part of thorax medium brown, sutures, apodemes and the shoulder spots dark brown. Length of fore wing 1.4-2.2 mm, of hind wing 1.4-1.7 mm. Pregenital part of abdomen light brown.

Male terminalia (Figs 9-13) dark brown, well sclerotized. Hypandrium as long as high in lateral view. Processus terminalis distinct but truncated obliquely in ventral view. 


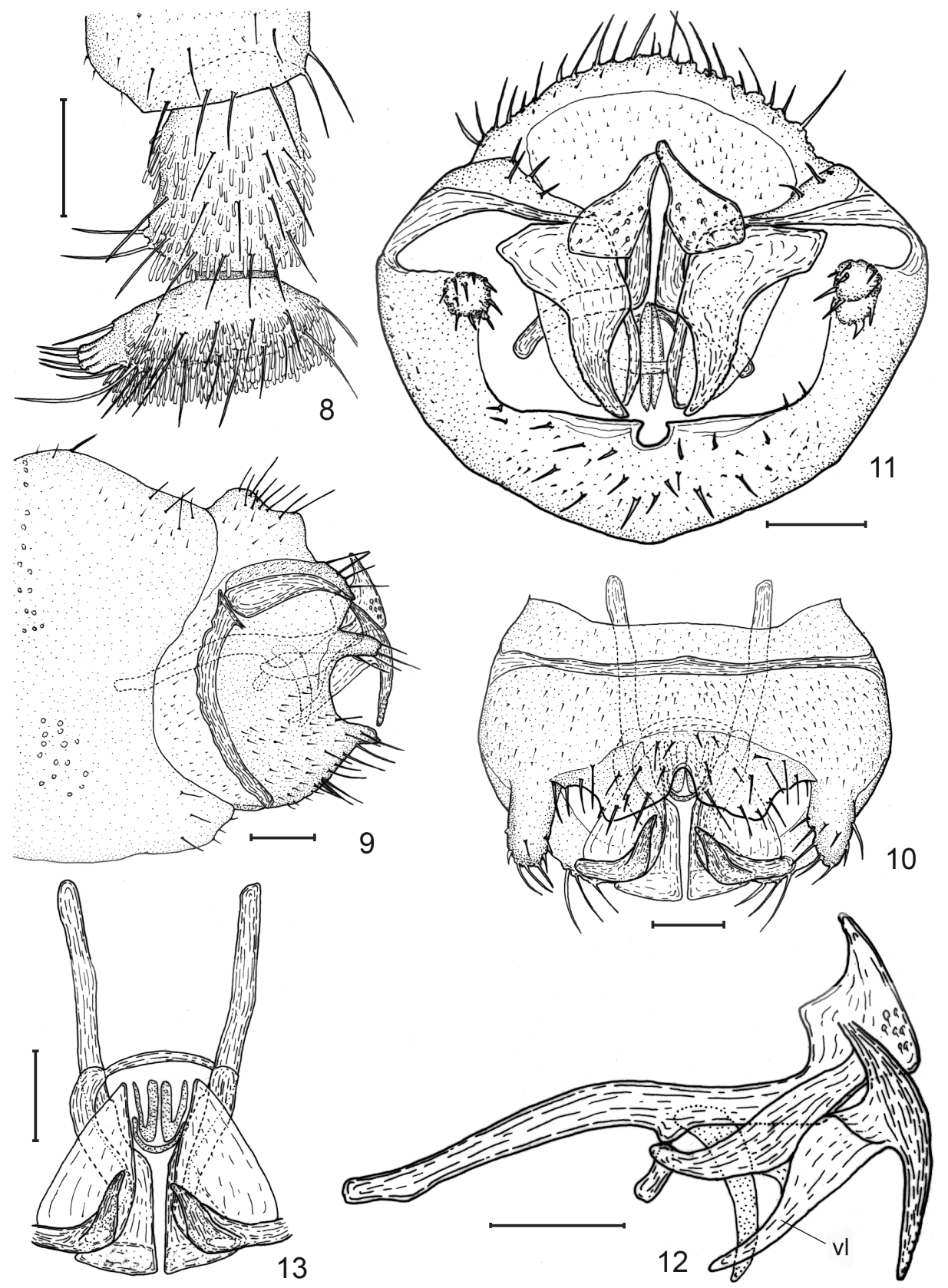

Figs 8-13. Coniopteryx (C.) evellana sp. n.: $8=$ basal part of antenna, $9=$ male terminalia, lateral view, $10=$ male terminalia, ventral view, $11=$ male terminalia, caudal view, $12=$ male internal genitalia, lateral view, $13=$ male internal genitalia, ventral view. Abbreviation: vl = ventral lobe of the inner branch of stylus. Scales: $0.04 \mathrm{~mm}$. 
Therefore, median incision consists of a very shallow " $\mathrm{V}$ " shaped part and of a narrow engraving in the middle of it. Processus lateralis small and directed caudally. Its setae are rather short. Anterior apodeme of hypandrium almost straight in ventral view, while in lateral view curved backwards (and stongly widened) dorsally. Gonarcus well sclerotized, with a broad basal part. Stylus forked. Its outer branch narrow in lateral, broad in caudal view, with a very finely serrated ending. Inner branch of stylus wide with a peculiar broad, plate-like, subtriangular ventral lobe. The ventral bridge between the two inner branches moderately sclerotized. Paramere moderately long; its caudal part turned upwards subapically in an obtuse angle. Processus ventralis small, but distinct. Processus apicalis rather small also, directed upwards, with a tooth-like upper and a plate-like lower part. The penis sclerite consists of two moderately chitinized, bent rods.

Remarks: Coniopteryx (C.) evellana belongs to Coniopteryx lobifrons group sensu SzIRÁKI (2005), and - regarding the processus terminalis, median incision and distal part of paramere - it resembles to Coniopteryx (C.) oroszi Sziráki, 2004 from Thailand.

The main distinctive features of the new species are:

- pedicel of antenna without a very strong seta (which is characteristic in C. oroszi);

- presence of the plate-like subtriangular lobe of the inner branch of the stylus;

- $\quad$ the inner branch of the stylus wide;

- the small processus lateralis of hypandrium directed caudally.

Etymology: The new species is named after the structure of median incision of hypandrium.

\section{Coniopteryx (Coniopteryx) malgasensis sp. $\mathrm{n}$.}

(Figs 14-19)

Coniopteryx (C.) madagascariensis sensu Meinander 1998, Figs 17A, 17D, 17F, 17H, 17J.

Examined material - Holotype: male, Madagascar, Antanarivo Province, $3 \mathrm{~km} \mathrm{NE}$ of Andranomay, $18^{\circ} 28^{\prime} 24^{\prime \prime}$ S, 47 57'36" E, 1300 m. a.s.l., mountain rainforest, 5-13. XII. 2000, Malaise trap, leg.: F, G et al.; deposited in the collection of CAS. Paratypes: 1 male, Madagascar, Antanarivo Province, $7 \mathrm{~km}$ SE of Andasibe National Park headquarters, $18^{\circ} 57^{\prime} 46^{\prime \prime} \mathrm{S}$, $48^{\circ} 27^{\prime} 10^{\prime \prime}$ E, $1050 \mathrm{~m}$ a.s.l., tropical forest, 7-22. I. 2001, Malaise trap, leg.: HH; 2 males, same data but 23. III - 7. IV. 2001; 3 males, same data but 9-23. IV.2001; 1 male, Madagascar, Antanarivo Province, botanic garden near to the entrance to Andashibe National Park, $18^{\circ} 55^{\prime} 39^{\prime \prime}$ S, 48 $24^{\prime} 28^{\prime \prime}$ E, $1025 \mathrm{~m}$ a.s.l., tropical forest, 14-21. V. 2001, Malaise trap, leg.: HH; 1 male, same locality, but 2-8. X. 2001, leg.: I \& HH; 1 male, same data as holotype; 1 male, Madagascar, Antsiranana Province, Sakalawa Beach, $12^{\circ} 15^{\prime} 46^{\prime \prime}$ S, 49 $23^{\prime} 51^{\prime \prime}$ E, $10 \mathrm{~m}$ a.s.l., dwarf littoral forest, 13-16. V. 2001, Malaise trap, leg.: HH; 2 males, same data but 25. VI - 6. VII. 2001; 1 male, same data but 7-22. VII. 2001; 2 males, same data but 28. VIII - 12. IX. 2001; 2 males, Madagascar, Diego - Suarez Province, Montagne d'Ambre National Park, 12 $30^{\prime} 52^{\prime \prime}$ S, 49¹0'53" E, 960 m a.s.l., 21-26. I. 2001, Malaise trap, leg.: I, ES \& HH; 1 male, same data 
but 4-19. III. 2001, leg.: HH; 1 male, same data but 19. III - 5. IV. 2001; 8 males, Madagascar, Diego - Suarez Province, Montagne d'Ambre National Park, 12³1' S, 49¹1' E, 975 m a.s.l., 21-25. I. 2001, Malaise trap, leg.: I, ES \& HH; 2 males, same data but 25-29. I. 2001; 1 male, same data but 4-19. III. 2001, leg.: HH; 1 male, Madagascar, Diego - Suarez Province, Mon-

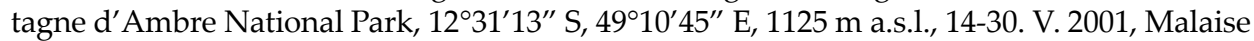
trap, leg.: HH; 2 males, Madagascar, Diego - Suarez Province, 7 km N of Joffreville, $12^{\circ} 20^{\prime} \mathrm{S}$, 49 $9^{\circ} 5^{\prime}$ E, 360 m a.s.l., dry forest, 6-20. III. 2001, Malaise trap, leg.: HH; 2 males, Madagascar, Diego - Suarez Province, Montagne des Français, $12^{\circ} 18^{\prime} 08^{\prime \prime}$ S, 49 $38^{\prime} 51^{\prime \prime}$ E, 150 m a.s.l., forested limestone ridge, 30. I - 15. II. 2001, Malaise trap, leg.: HH; 1 male, same data but 6-20. III. 2001; 6 males, Madagascar, Fianarantsoa Province, Ranomafana National Park, Belle Vue at Talatakely, $21^{\circ} 15^{\prime} 59^{\prime \prime}$ S, $47^{\circ} 25^{\prime} 13^{\prime \prime}$ E, $1020 \mathrm{~m}$ a.s.l., secondary tropical forest, 8-15. XI. 2001, Malaise trap, leg.: HH; 6 males, same data but 15-22. XI. 2001; 1 male, same data but 28. XI - 6. XII. 2001; 1 male, same data but 6-15. XII. 2001; 9 males, same data but 24. XII. 2001 2. I. 2002; 1 male, same data but 10-14. I. 2002; 1 male, same data but 14-21. I. 2002; 9 males, same data but 21-28. I. 2002; 1 male, same data but 28. I - 4. II. 2002; 1 male, same data but 4-12. II. 2002; 1 male, same data but 12-19. II. 2002; 3 males, same data but 31. III - 7. IV. 2002; 3 males, same data but 7-14. IV. 2002; 3 males, same data but 14-23. IV. 2002; 3 males, same data but 13-23. V. 2002; 2 males, same data but 23. V - 3. VI. 2002; 2 males, same data but $13-$ 23. VI. 2002; 2 males, same data but 4-14. VII. 2002; 1 male, same data but 14-24. VII. 2002; 2 males, same data but 24 . VII - 4. VIII. 2002; 6 males, same data but 16 . X-8. XI. 2002; 9 males, same data but 22-28. XI. 2002; 1 male, same data but 28. V - 6. VI. 2003; 2 males, Madagascar, Fianarantsoa Province, Ranomafana National Park, Jirama water works, $21^{\circ} 14^{\prime} 55^{\prime \prime}$ S, $47^{\circ} 27^{\prime} 08^{\prime \prime}$ E, $690 \mathrm{~m}$ a.s.l., open area near a stream, 16. X - 8. XI. 2001, Malaise trap, leg.: HH; 1 male, same data but 22-28. XI. 2001; 1 male, same data but 28. XI - 6. XII. 2001; 5 males, same data but 21-24. XII. 2001; 1 male, same data but 21-28. I. 2002; 4 males, Madagascar, Fianarantsoa Province, Ranomafana National Park, radio tower, $21^{\circ} 15^{\prime} 30^{\prime \prime}$ S, $47^{\circ} 24^{\prime} 26^{\prime \prime}$ E, $1130 \mathrm{~m}$ a.s.l., mixed tropical forest, 8-15. X. 2001, Malaise trap, leg.: HH; 3 males, same data but 16. X-8. XI. 2001; 1 male, same data but 15-22. XI. 2001; 1 male, same data but 22-28. XI. 2001; 10 males, same data but 28. XI - 6. XII. 2001; 2 males, same data but 6-15. XII. 2001; 5 males, same data but 15-21. XII. 2001; 1 male, same data but 24. XII. 2001 - 2. I. 2002; 2 males, same data but 10-14. I. 2002; 5 males, same data but 14-21. I. 2002; 3 males, same data, but 21-28. I. 2002; 2 males, same data but 12-19. II. 2002; 1 male, same data but 4-12. III. 2002; 1 male, same data but 12-19. III. 2002; 1 male, same data but 26-31. III. 2002; 2 males, same data but 9-16. IV. 2002; 1 male, same data but 16-23. IV. 2002; 2 males, same data but 23-30. IV. 2002; 3 males, same data but 24 . V - 4. VI. 2002; 1 male, same data but 14-24. VI. 2002; 3 males, same locality, but 15-25. VII. 2002, leg.: I \& HH; 2 males, same data but 25. VII - 5 . VIII. 2002; 1 male, same data but 6-17. VII. 2003; 1 male, Madagascar, Fianarantsoa Province,

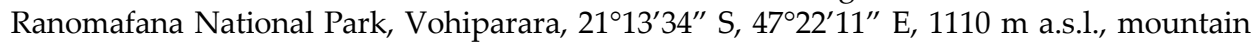
rainforest, 16. X - 8. XI. 2001, Malaise trap, leg.: HH; 1 male, same data but 15-22. XI. 2001; 3 males, same data but 22-28. XI. 2001; 18 males, same data but 28. XI - 6. XII. 2001; 3 males, same data but 6-15. XII. 2001; 3 males, same data but 15-21. XII. 2001; 1 male, same data but 24. XII. 2001 - 2. I. 2002; 1 male, same data but 2-10. I. 2002; 2 males, same data but 14-21. I. 2002; 3 males, same data but 21-28. I. 2002; 12 males, same data but 28. I - 4. II. 2002; 2 males, same data but 4-12. II. 2002; 2 males, same data but 19-26. II. 2002; 1 male, same data but 8-15. IV. 2002; 1 male, same data but 15-22. IV. 2002; 1 male, same data but 22-29. IV. 2002; 1 male, same data but 6-15. V. 2002; 1 male, same data but 15-25. V. 2002; 1 male, same data but 5-15. VII. 2002; 3 males, same data but 15-25. VII. 2002; 2 males, Madagascar, Fianarantsoa Province, Ranomafana National Park, Talatakely, $21^{\circ} 15^{\prime} 30^{\prime \prime}$ S, $47^{\circ} 25^{\prime} 28^{\prime \prime}$ E, 900 m a.s.l. 28. IV. 1998, leg.: G, K, P, MR, JR, JS, U. Majority of paratypes are deposited in the collection of 

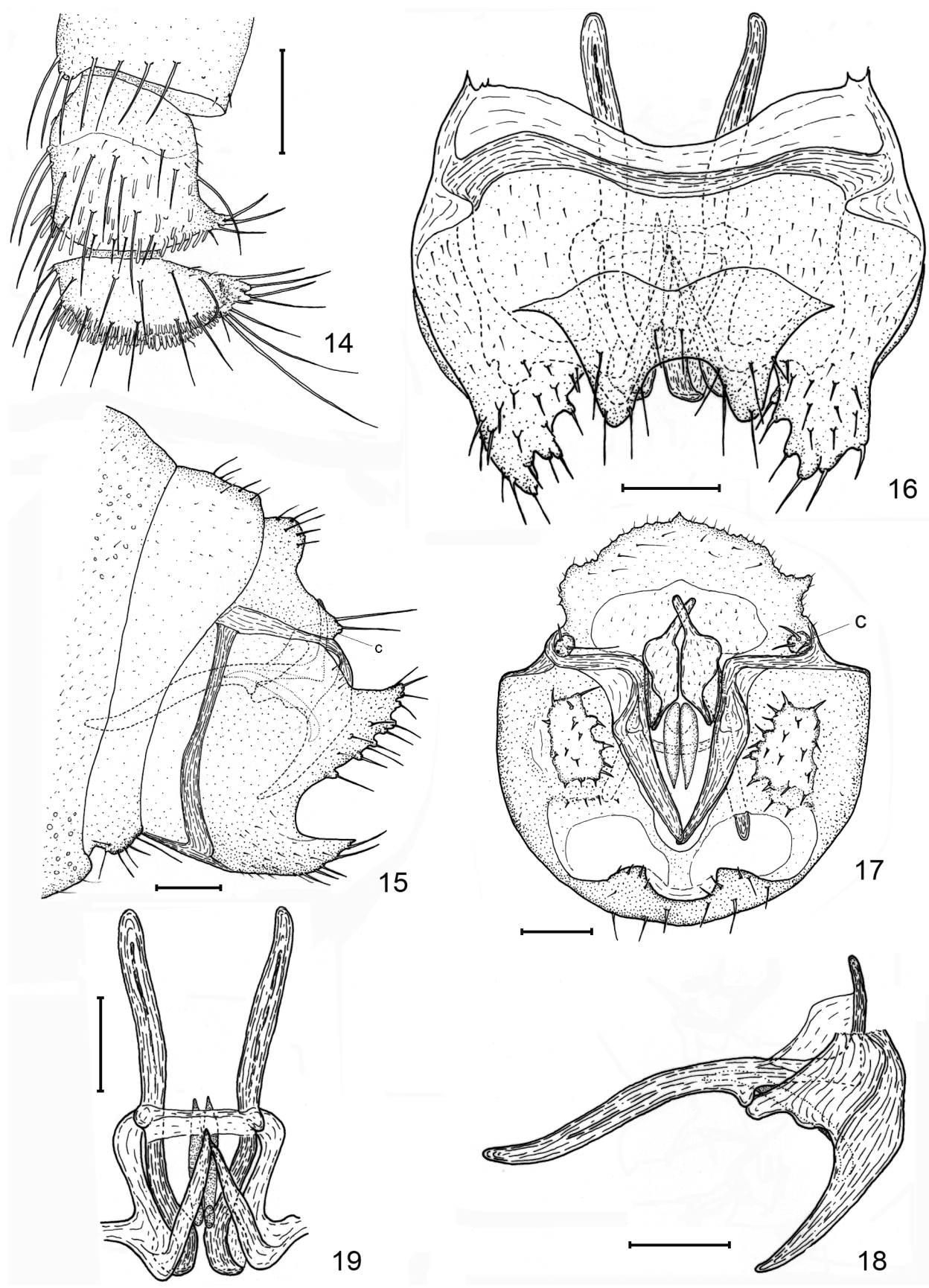

Figs 14-19. Coniopteryx (C.) malgasensis sp. $n .: 14=$ basal part of antenna, 15-17: = male terminalia, 15 = lateral view, 16 = ventral view, $17=$ caudal view, 18-19= male internal genitalia, $18=$ lateral view, $19=$ ventral view. Abbreviation: $\mathrm{c}=$ caudal projection of gonarcus. Scale: $0.04 \mathrm{~mm}$. 
California Academy of Sciences, while 6 paratype specimens are housed in the Hungarian Natural History Museum, Budapest.

Diagnosis: Small bodied coniopterygid, with flattened projection between the antennae. Pedicel and first flagellar segment with setose outgrowth. Median incision of processus terminalis u-shaped. Setae of processus lateralis with prominent bases.

Description: Length of the body 1.4-1.9 mm. Head capsule, palpi and scape light brown, pedicel and flagellum medium brown. Between the antennae the anterior edge of vertex has a prominent, laterally flattened projection. Eyes moderately large, black. Antennae $0.9-1.1 \mathrm{~mm}, 26-28$ segmented. Scape as long as wide, pedicel 1.2 times longer than wide, basal flagellar segments almost two times, median ones 1.5 times as wide as long. Pedicel has a smaller, while the first flagellar segment has a larger setose outgrowth apically (Fig. 14). Setae of the basal flagellar segments are about as long as width of the segments, while those of the median and apical segments shorter. Ordinary hairs are arranged in two rather irregular rings on flagellar segments. Scale-like hairs on the whole surface of the pedicel, and in a dense apical whorl on flagellar segments.

Legs and large part of thorax light brown, sutures, apodemes and the shoulder spots medium or dark brown. Length of fore wing 1.4-1.9 mm, of hind wing 1.4-1.6 mm. Wing membrane and veins light or medium brown. Pregenital part of abdomen whitish.

Male terminalia (Figs 15-19) well sclerotized. Hypandrium as long as high in lateral view. Tips of the processus terminalis rounded in ventral view. Median incision moderately deep, " $\mathrm{u}$ "-shaped. Dorso-caudal ending of processus lateralis acute, with prominent bases of the relatively short setae. Anterior apodeme of hypandrium wide, and curved backwards medially. Gonarcus with small, setose caudal projection. Stylus forked; its outer branch moderately wide, acute, while the inner branch very wide. The bridge between the two inner branches slightly sclerotized. Processus ventralis of paramere distinct. Processus apicalis moderately large, pointed, directed upwards, has an acute ventro-caudal angle, and there are a larger dorsal and a smaller ventral thin plate between this process and the stem of the paramere. The penis sclerite consists of two moderately chitinized, acute, bent and caudally slightly hooked rods.

Remarks: Coniopteryx (C.) malgasensis belongs to Coniopteryx lobifrons group sensu SzIRÁкI 2005, and it is surprisingly close to Coniopteryx (C.) alticola Sziráki, 2002 from Thailand.

The main distinctive features of the new species are:

- pedicel of antenna has a small setose outgrowth (not only the first flagellar segment);

- caudal projection of gonarcus small;

- anterior apodeme of hypandrium curved backwards medially;

- setae of dorso-caudal ending of processus lateralis short, with prominent bases.

Etymology: The new species is named after an earlier name (Malagasy Republic) of Republic of Madagascar. 


\section{DISCUSSION}

Two of the described new species - determined by Meinander (1998) as variations of $C$. madagascariensis - were represented by a really large number of specimens in the examined material. Namely, 384 specimens proved to be $C$. evellana sp. $\mathrm{n}$. and 237 to C. malgasensis sp. $\mathrm{n}$. All of these specimens were determinable, there was not any transitorial form. Consequently, it may be stated that the intraspecific variability is not larger in these cases, as in other (e. g. European) species of the genus Coniopteryx. Therefore, the three kind of male genitalia figured by MeinANDER (1998, Figs 17A-K) represents three different species, and none of them is equal with C. madagascariensis. It is clear already on the basis of the examination of genitalia. Moreover, investigation of the holotype of C. madagascariensis, and the newly collected specimen conspecific with it, showed that the original description of the $C$. madagascariensis - contrary to the statement of Meinander (1998) - was correct. That is, in this case there are no frontal projection on the vertex and setose outgrowth on the first flagellar segment, which characters are shared in all new species described in present paper, and - in general - in the case of the C. lobifrons species group. Consequently, Coniopteryx madagascariensis Meinander, 1974 - belonging to the Coniopteryx notata species group (SzIRÁKI 2005) - not simply differs from the three species, which were later handled by MeinANDer (1998) as C. madagascariensis, but seems to be rather far from those within the subgenus Coniopteryx. Finally, it is worth to mention that the Coniopteryx lobifrons species group hitherto was known only from the Oriental Region. This interesting widening of the known geographical distribution will be discussed in a following paper.

Acknowledgements - I am indebted to Dr. Norman D. Penny for the possibility to examine this extremely interesting Madagascan coniopterygid material, as well as to Dr. Jean Legrand, Muséum National d'Histoire Naturelle (Paris) for the kind loan of the holotype of Coniopteryx madagascariensis.

\section{REFERENCES}

Meinander, M. (1974) Coniopterygidae from Madagascar (Neuroptera). Notulae Entomologicae 54: 60-63.

Meinander, M. (1998) Coniopterygidae (Neuroptera) from southern and eastern Africa. African Entomology 6: 117-146.

SzIrákI, Gy. (2005) Species grouping of several genera and subgenera of Coniopterygidae (Neuroptera). Folia entomologica hungarica 66: 101-136.

Revised version received November 12, 2014, accepted March 30, 2015, published May 29, 2015 